\title{
Correction: Cisplatin-induced epigenetic activation of miR-34a sensitizes bladder cancer cells to chemotherapy
}

Heng $\mathrm{Li}^{1 \dagger}$, Gan Yu${ }^{1 \dagger}$, Runlin Shi ${ }^{1}$, Bin Lang ${ }^{3}$, Xianguo Chen ${ }^{4}$, Ding Xia ${ }^{1}$, Haibing Xiao ${ }^{1}$, Xiaolin Guo ${ }^{1}$, Wei Guan ${ }^{1}$, Zhangqun $\mathrm{Ye}^{1}$, Wei Xiao ${ }^{2^{*}}$ and Hua Xu ${ }^{1^{*}}$

\section{Correction}

After the publication of this work [1] it was brought to the authors' attention that Figures six (Figure 1 here) (E) and (F) contained an error in their data presentation. The correct figure is given below.

We regret any inconvenience that this inaccuracy may have caused.

\footnotetext{
* Correspondence: xiaowei0041@163.com; xuhua@mail.hust.edu.cn

${ }^{\dagger}$ Equal contributors

${ }^{2}$ Translational Medicin Center, Tongji Hospital, Tongji Medical College,

Huazhong University of Science and Technology, Wuhan 430030, China

${ }^{1}$ Department of Urology, Tongji Hospital, Tongji Medical College, Huazhong

University of Science and Technology, Wuhan 430030, China

Full list of author information is available at the end of the article
} 


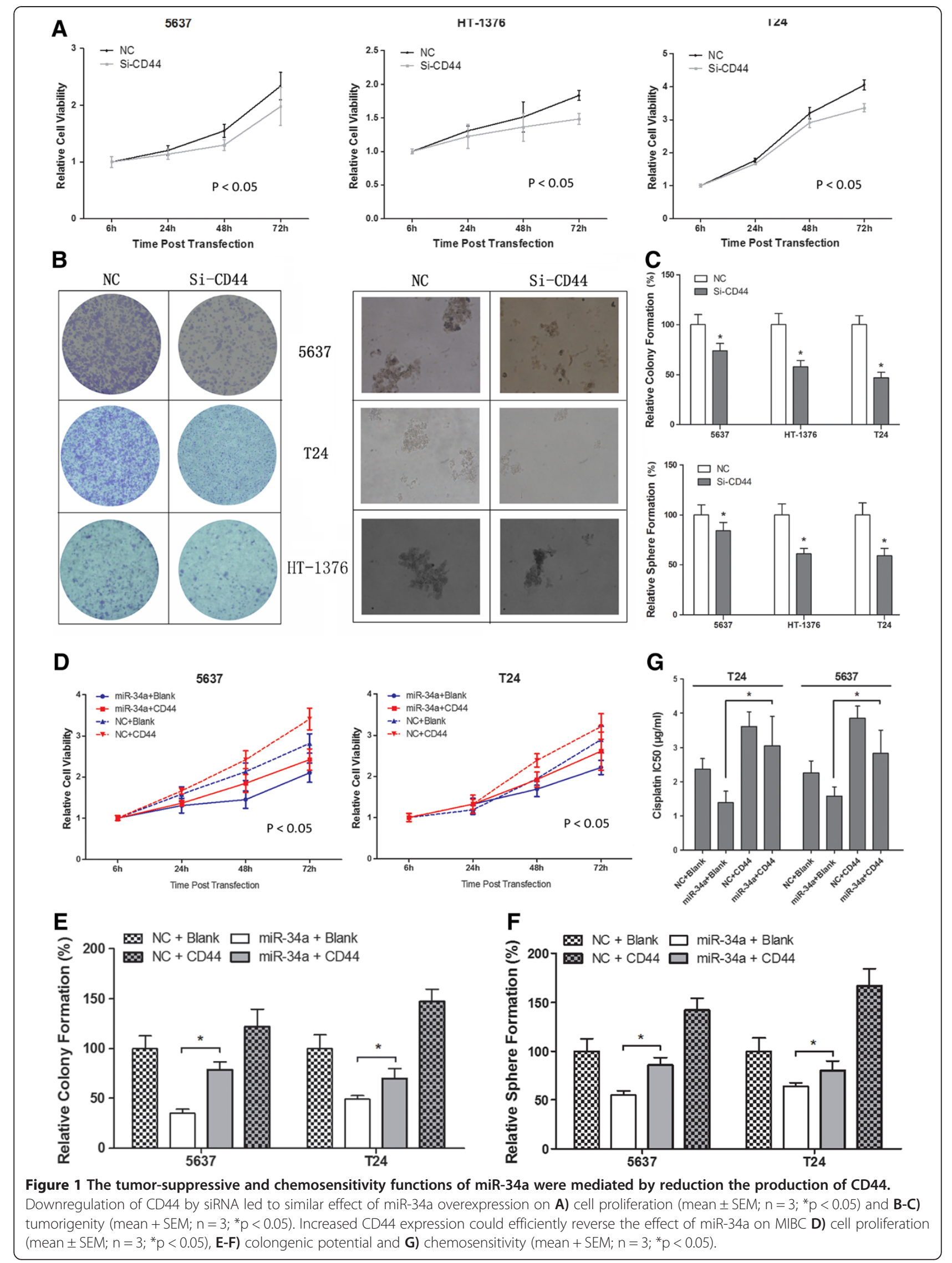




\section{Author details}

${ }^{1}$ Department of Urology, Tongji Hospital, Tongji Medical College, Huazhong University of Science and Technology, Wuhan 430030, China. ${ }^{2}$ Translational Medicin Center, Tongji Hospital, Tongji Medical College, Huazhong University of Science and Technology, Wuhan 430030, China. ${ }^{3}$ School of Health

Sciences, Macao Polytechnic Institute, Macao, China. ${ }^{4}$ Department of Urology,

First Affiliated Hospital of Anhui Medical University, Hefei, Anhui 230022,

China.

Received: 5 August 2014 Accepted: 5 August 2014

Published: 14 August 2014

\section{Reference}

1. Heng L, Gan Y, Runlin S, Bin L, Xianguo C, Ding X, Haibing X, Xiaolin G, Wei $G$, Zhangqun $Y$, Wei $X$, Hua $X$ : Cisplatin-induced epigenetic activation of miR-34a sensitizes bladder cancer cells to chemotherapy. Molecular Cancer 2014, 13:8.

doi:10.1186/1476-4598-13-183

Cite this article as: Li et al:: Correction: Cisplatin-induced epigenetic activation of miR-34a sensitizes bladder cancer cells to chemotherapy. Molecular Cancer 2014 13:183. 